Forschungsassitentin

Forschungsstelle für Rechtsquellenerschließung der Universität Wien

Österreichische Akademie der Wissenschaften

\title{
Der Vertrag von Saint Germain im Kontext der europäischen Nachkriegsordnung Internationale Konferenz (Wien, 27.-29. September 2018)
}

\section{The Treaty of Saint Germain 1919 in the Context of the European Post-War Order International Conference (Vienna, September 27 $7^{\text {h }}-29^{\text {th }}$ 2018)}

In view of the approaching centenary of the Paris Peace Conference 1919/1920 and the conclusion of the so-called "Parisian Peace Treaties" terminating the First World War, an interdisciplinary international conference organized by the Austrian Academy of Science took place in Vienna from September 27 th till 29th 2018 . With a view on the Austrian Treaty, the Treaty of Saint Germain, and its consequences it attempted to shed light on the developments and parties involved in the broader context of the European Post-War Order, thereby revealing the large and varied scope of the provisions as well as several research desiderata.

Keywords: Paris Peace Treaties, Treaty of Saint Germain, interwar period, Europe

\section{Einführung und Überblick}

Angesichts des bald hundertjährigen Bestehens der sogenannten Pariser Vororteverträge, die im Gefolge der Pariser Friedenskonferenz 1919/1920 unterzeichnet wurden und aus juristischer Sicht das Ende des Ersten Weltkriegs markieren, wurde Ende September in Wien eine Internationale Konferenz veranstaltet. Als Veranstaltungsträger fungierte die Österreichische Akademie der Wissenschaften in Form des Instituts für Neuzeit- und Zeitgeschichtsforschung (INZ) sowie der Kommission für die Rechtsgeschichte Österreichs (KRGÖ) in Kooperation mit der Forschungsstelle für Rechtsquellenerschließung der Universität Wien (FRQ); für die Organisation zeichneten Michael Gebler (Universität Hildesheim), Thomas Olechowski (Universität Wien), Stefan Wedrac (ÖAW) sowie Anita Ziegerhofer (Universität Graz) verantwortlich.

Mit dem zwischen den alliierten und assoziierten Mächten und der Republik Österreich am 10. September 1919 unterzeichneten Vertrag von Saint-Germain-en-Laye wurde zum einen bewusst einer der im Vergleich zum Versailler Vertrag mit Deutschland weniger bekannten Verträge als Ausgangspunkt gewählt. Die einzelnen Beiträge sollten Entstehung und Wirken des Vertrages im Gesamtkontext der Vororteverträge und der neuen internationalen Ordnung beleuchten, wobei der Fokus gleichermaßen auf Siegermächten wie Verlierern und neu entstandenen Staaten liegen sollte. In den Vordergrund gerückt wurde dabei zum anderen weniger die - vergleichsweise besser aufgearbeitete - Entstehungsgeschichte des Vertrags auf der Pariser 
Friedenskonferenz, sondern die Konzentration auf die Entwicklungen in der Zwischenkriegszeit und die Auswirkungen der Regelungen im Zusammenhang mit der Neuordnung Europas. Diesen beiden Aspekten versucht ein seit 2017 an der KRGÖ in Kooperation mit dem INZ und den Universitäten Graz, Linz und Wien laufendes Forschungsprojekt zur rechtlichen Bedeutung des Vertrags von St. Germain Rechnung zu tragen, dessen Forschungen durch den Austausch und die Vernetzung auf der Konferenz in die internationale Forschungslandschaft eingebettet werden können. Insgesamt sollte die Aufmerksamkeit auch auf bislang in diesem Zusammenhang kaum erforschte Regelungsbereiche, etwa auf wirtschaftlichem und kulturellem Gebiet, gelenkt und ein neuer Blick auf die Gesamtbewertung der Pariser Friedensordnung ermöglicht werden.

Dementsprechend gliederte sich das dreitägige Programm in sechs Panels: Nach einer Einführung in den europäischen und internationalen Kontext durch die ersten beiden Vorträge sollten sich die darauffolgenden jeweils auf eine der fünf „alliierten und assoziierten Hauptmächte“ (Frankreich, Großbritannien, die USA, Italien und Japan) konzentrieren. Das dritte und letzte Panel des ersten Tages rückte den Blick auf nach dem Kriegsende neu entstandene Staaten, die Tschechoslowakei, den SHS-Staat und Polen. Am Freitag standen zunächst die Konsequenzen des Vertrags von St. Germain für das Österreich der Zwischenkriegszeit im Mittelpunkt. Beginnend mit den Auswirkungen auf die österreichische Bundesverfassung 1919/20 und der Institutionalisierung seiner Ausführung in Form von Vertragsorganen befassten sich die weiteren Vorträge jeweils mit bestimmten Bereichen, vom finanziellen über den militärischen bis hin zu Kulturgüterrückführung und Verkehrswesen. Der Nachmittag stand bereits im Zeichen des vorletzten Panels, der Pariser Nachkriegsordnung und den übrigen Verliererstaaten. Auf zwei Vorträge zum Versailler Vertrag folgten Präsentationen zum Vertrag von Trianon mit Ungarn und von Neuilly mit Bulgarien. Der dritte Tag begann mit zwei Beiträgen zu den Verträgen von Sévres 1920 und Lausanne 1923 mit dem Osmanischen Reich bzw. der Türkei. Das sechste und letzte Panel wandte sich schließlich wieder vom Speziellen zum Allgemeinen, indem die verbleibenden drei Vorträge die Position des Vatikans, die Festlegung von Staatsgrenzen und die völkerrechtlichen Konsequenzen herausstellen sollten.

\section{Begrüßung und Eröffnung}

Eröffnet wurde die Konferenz durch die Begrüßungsworte der Vertreter zweier Veranstalter, Katrin Keller und Thomas Olechowski. Keller, Direktorin des Instituts für Neuzeit- und Zeitgeschichtsforschung, drückte ihre Freude über das Zustandekommen der Konferenz und die Kooperation der verschiedenen Veranstalter aus; ihre guten Wünsche für die Veranstaltung und deren Beitrag zu weiteren Forschungen begleiteten die darauffolgenden Tage. Olechowski, Obmann der KRGÖ an der ÖAW und Leiter der FRQ, bereitete mit seinem Rückblick auf den Wiener Kongress, der letzten großen Zusammenkunft zur Neuordnung Europas etwa 100 Jahre vor der Pariser Friedenskonferenz, und dem Ausblick auf die weitere völkerrechtliche Entwicklung bereits den Boden für die Einführung in den Gesamtkontext im ersten Panel, wobei er insbesondere auch die Frage in den Raum stellte, ob die völkerrechtlichen Impulse der Friedensverträge nicht in gewisser Weise ein bereits in den Vorkriegsjahren entstandenes Aufstreben fortsetzten. 


\section{Kontextuelle Einführung}

Den Auftakt bildeten die fulminanten, ebenso breiten wie tiefgehenden Vorträge zweier Mitglieder des Organisationsteams, Michael Gebler und Anita Ziegerhofer.

Gebler (Hildesheim - Wien) hob zu Beginn die zahlreichen geschichtstheoretischen und methodischen Fragen der Thematik hervor, zumal es unmöglich sei, den Staatsvertrag - in zeitlicher wie in geographischer Hinsicht - isoliert zu betrachten. In seinem Vortrag spannte er in sieben Punkten den Bogen vom Sommer 1914, als der Kriegsausbruch nach Clark die ersehnte „Befreiung aus Langeweile“ brachte, bis hin zum Beginn einer neuen Ära, als sich ein Wandel im Zeichen der Entmachtung Europas abzeichnete. Aus dem Grundsatzkonflikt seien keine wirklichen Sieger hervorgegangen; das Ziel einer Befriedung nicht erreicht worden. Die Verhandlungen hätten in Anbetracht der Überforderung der Beteiligten und allen Bemühungen zum Trotz in ein Diktat gemündet und eine Neudefinition von Europa war gescheitert. Gegen bzw. mit dem Ende des Weltkriegs waren nicht weniger als 13 neue Staaten entstanden und vier Großreiche zusammengebrochen; die neuen Grenzen zogen eine Fülle neuer Probleme nach sich. Neben den politischen Umwälzungen war es zu Brüchen auf gesellschaftlicher Ebene gekommen. So hatten sich in den Jahren von 1917 - 1920 entscheidende Veränderungen von welthistorischer Bedeutung vollzogen, die das gesamte darauffolgende Jahrhundert prägen sollten und zum Teil bis in unsere Zeit hineinreichen.

Gleich im Anschluss gelang es Ziegerhofer (Graz) in rund 30 Minuten eine auf das Wesentliche reduzierte Geschichte der beiden internationalen Organisationen zu erzählen, deren Satzungen die gemeinsame Klammer der Friedensverträge bildeten und die Vision vom politischen und sozialen Weltfrieden verkörperten: der Völkerbund und die Internationale Arbeitsorganisation (ILO). Ausgehend von wesentlichen Faktoren, die vor dem Hintergrund einer neuen internationalen Öffentlichkeit, der Friedensbewegung und des Krieges für die Gründung des Völkerbunds als erste globale Internationale Organisation eine Rolle spielten, bot sich zuerst ein umfassender Überblick über die variierende Mitgliederanzahl (neben dem Fernbleiben der USA waren 17 Austritte, 22 Beitritte und ein Ausschluss zu verzeichnen), seine Organstruktur und Tätigkeitsfelder, dar. Daraufhin folgte ein Einblick in die Entstehungsgeschichte der organisatorisch mit dem Völkerbund verbundenen ILO aus einem Kompromiss zwischen Arbeiterbewegung, Regierungen und der Industrie; Jene wies mit der dreiteiligen Besetzung von Gremien, der besonderen Berücksichtigung von Frauen und der Möglichkeit weisungsungebundener Handlungen auf internationaler Ebene einige Besonderheiten auf und entfaltete rasch eine rege Tätigkeit. Seit dem Beitritt der USA 1934 wurde ihre Satzung als „Verfassung“ bezeichnet. Die nachträgliche Bewertung der beiden Organisationen steht in starkem Kontrast: Dem Erfolg der bis heute bestehenden ILO wird das Scheitern des Völkerbunds gegenüber gestellt, wiewohl auch dieser in einzelnen Bereichen, wie etwa dem Minderheitenschutz, durchaus eine konstruktive Politik entwickelt hatte. 


\section{Die Pariser Nachkriegsordnung und die Siegermächte}

In diesem Panel übernahmen die Vortragenden den Versuch, die „klassische“ Fokussierung auf die Politik der einzelnen Siegermächte, prominent verkörpert durch die später als „Big Three“ bezeichneten Staatschefs Wilson, Lloyd George und Clémenceau, in Bezug auf den Vertrag von St. Germain zu ergänzen. Der Vortrag zu Italien widmete sich hingegen mit der Tiroler Frage einer ebenfalls klassischen, allerdings Saint Germain spezifischen Thematik, während die Präsentation zur Tätigkeit der Delegierten Japans in den Grenzziehungskommissionen leider ausfiel.

Den Anfang machte mit Catherine Horel (Paris) eine ausgewiesene Kennerin von ungarischen und zentraleuropäischen Fragestellungen in diesem Zusammenhang. Sie betonte, dass die französische Österreichpolitik Frankreichs vor dem Krieg mitnichten auf eine Zerstückelung der Habsburgermonarchie gerichtet war und erst durch die Beteiligung am Londoner Vertrag ein explizit gegen die Monarchie gerichtetes französisches Kriegsziel bestand. Nach dem Krieg und dem Zusammenbruch standen - vor dem Hintergrund eines möglichen Anschlusses an ein dadurch erstarkendes Deutschland - die Frage der Rechtsnachfolge und der künftigen Stellung des neuen österreichischen Staates (Stichwort: Unabhängigkeit) im Zentrum; nach dem Abschluss des Vertrags prägten schließlich die Angst vor dem Bolschewismus und dem deutschen Revanchismus die französische Politik. In der darauffolgenden Diskussion wurde vor allem Clémenceaus Österreichbild und die Wahrnehmung des deutsch-österreichischen Verhältnisses thematisiert.

Im darauffolgenden Beitrag von B.J.C. McKercher (Victoria) konnten sich die Teilnehmenden ein Bild von Lloyd George und der britischen Position in Bezug auf das neue Österreich machen, wobei der Vortragende in der Diskussion die Unterschiede zwischen den Vorstellungen des Foreign Office und des Premierministers abermals betonte. Die Briten selbst hatten zunächst keine Vorstellung davon, wie Mitteleuropa nach dem Krieg aussehen sollte; Österreich war für Lloyd George, für den die Anschlussfrage kaum eine Rolle spielte, nur einer von mehreren Nachfolgestaaten, denen er insgesamt eher skeptisch gegenüberstand, wobei seine Politik insgesamt eher von pragmatischen als strategischen Überlegungen getragen war. Nach seiner Abreise prägten freilich vor allem Balfour und Eyre Crowe die britische Österreichpolitik, die durch ein Bemühen um Stabilisierung, nicht zuletzt in finanzieller Hinsicht geprägt war: Österreich sollte ein demokratischer, kapitalistischer (und letzten Endes auch zahlungspflichtiger) Staat werden.

Hans-Jürgen Schröder (Gießen) übernahm es in seiner konzisen Darstellung, den Staatsvertrag von St. Germain in den Kontext der imperialen Bestrebungen der USA einzubetten. Auch die USA verfolgten zunächst keine unmittelbaren Interessen in Bezug auf die Monarchie, die Kriegserklärung war aus mehreren Gründen, darunter auch aus der Überzeugung heraus, dass Wien in einem Abhängigkeitsverhältnis zu Berlin stünde, erfolgt. Der österreichische Friedensvertrag ist einerseits in Bezug auf das von Wilson proklamierte Selbstbestimmungsrecht, als „Seismograph“ für seine Selbstbestimmungspolitik, andererseits aus vergleichender Perspektive zur deutschen Situation, was etwa die Demokratisierung der politischen Systeme, die territorialen Verluste und wirtschaftlichen Folgen anlangt, von Interesse. Beide Friedensverträge stellen sich letzten Endes als Etappe der us-amerikanischen Hegemonialpolitik dar; die USA sicherten sich schließlich in den Separatfrieden 1921 jeweils die Rechte aus diesen Verträgen - ohne Übernahme der Verpflichtungen. Aus der Diskussion ist der Vergleich zur vertraglichen Vernetzung nach dem 2. 
Weltkrieg sowie die Annahme, dass Wilson wohl nach seiner Rückkehr bereits nicht mehr an Österreich gedacht hat, hervorzuheben.

Den Abschluss des Panels bildete eine eingehende Darstellung der Tiroler Frage, die Stefan Wedrac (Wien) mit Blick auf das Zustandekommen der Entscheidung über die Teilung Tirols und Marion Dotter (Wien) im Hinblick auf deren Verwirklichung und Auswirkungen schilderten. Italien hatte die Brennergrenze bereits 1915 als klares Ziel formuliert und dafür Sorge getragen, dass sie auch Niederschlag in den militärischen Bestimmungen des Waffenstillstands finden sollte. Die schwierige Position Italiens in Paris verstärkte die Bedeutung dieser Frage, wobei die eigentliche Entscheidung bereits vor Ankunft der deutschösterreichischen Friedensdelegation fallen sollte; überraschend erscheint, dass Präsident Wilson hier keine Rücksicht auf eine mögliche Anwendung des Selbstbestimmungsrecht nahm, sondern gleichsam ,über Nacht“ entschied, die vorgeschlagene Regelung zu akzeptieren. Für die lokale Bevölkerung sollte diese Entscheidung einen gravierenden Einschnitt bedeuten und die Zwistigkeiten über die Stellung Südtirols noch weit in das 20. Jahrhundert hineinreichen.

\section{Neue Staaten und die Pariser Nachkriegsordnung}

Den Abschluss des ersten Tages bildete ein kurzes Panel zu Themen mit Bezug auf drei der zahlreichen neu entstandenen Staaten. Hier fand die Abgrenzungsfrage mit der „Kärntner Frage“ ihre Fortsetzung, während in den anderen beiden Vorträgen das Werden des jeweiligen neuen Staates und seine Verknüpfung mit den Pariser Verhandlungen anschaulich wurde.

Dalibor Trublar (Linz) betonte in seinem Vortrag die gemeinsame Geschichte der Republik Österreich und der Tschechoslowakei, die jedoch ganz unterschiedlich wahrgenommen wurde und in der die gleichen Bezugspunkte oftmals gegensätzlich bewertet wurden. Der Vertrag von St. Germain selbst wurde in der Tschechoslowakei kaum rezipiert, da die Tschechoslowakei alle ihre Forderungen im Wesentlichen erfüllt sah. Diese historisch bis hin zur Schlacht am Weißen Berg zurückreichenden Gegensätze zwischen dem Selbstverständnis der späteren Tschechoslowakei und der „österreichischen“ Wahrnehmung wurde auch in der Diskussion näher thematisiert.

Im Anschluss rief Richard Lein (Wien) den Teilnehmern in seiner ausführlichen Darstellung die „Ereignisse an der südslawischen Front" und die Grenzziehung zum SHS-Staat in Erinnerung. Während die Steiermark „handstreichartig“ von einem ehemaligen General der k.u.k. Armee besetzt wurde, kam es in Kärnten zu „Abwehrkämpfen“, deren Bedeutung laut Lein jedoch in der österreichischen Geschichtsschreibung tendenziell überschätzt wird. Dass es in Kärnten bekanntlich zur Anordnung und Durchführung einer Volksabstimmung kam, die in weiterer Folge zum Verbleib der betroffenen Gebiete bei Österreich führen sollte, sei vielmehr auf die politische Konstellation zurückzuführen; auf Seiten der Kärntner Slowenen dürfte für deren Ausgang wohl das Überwiegen des wirtschaftlichen gegenüber dem nationalen Interesse den Ausschlag gegeben haben.

Zu guter Letzt erhielten die Teilnehmer durch die Präsentation von Damian Szymzak (Posen) Einblick in die Entstehung des unabhängigen polnischen Staates im Gefolge der Pariser Friedensordnung. Für Polen war freilich in erster Linie der Versailler Vertrag von Bedeutung, der Vertrag von St. Germain ist hingegen weitgehend unbekannt. 


\section{Konsequenzen für Österreich}

Die sechs Vormittagsbeiträge wandten sich unterschiedlichen Themen aus dem Umkreis des österreichischen Vertrages zu.

Auch die Republik (Deutsch-)Österreich zählte nach ihrem Selbstverständnis zu den auf dem Boden der Monarchie neu entstandenen Staaten und lehnte jede Rechtsnachfolge ab, konnte ihren Standpunkt in Paris jedoch nicht durchsetzen. Dieser Übergang und die Auswirkungen des Staatsvertrags auf die Bundesverfassung, die ein gutes Jahr nach Vertragsunterzeichnung in Kraft treten würde, und an der bereits parallel zu den Friedensverhandlungen in St. Germain gearbeitet wurde, war Gegenstand des Beitrags von Thomas Olechowski (Wien), der durch die oft vernachlässigte Verknüpfung der Erzählstränge ein anschauliches und umfassendes Bild dieses Zeitraums zu zeichnen verstand.

Der Beitrag der Autorin hatte zum Ziel, die Aufmerksamkeit auf einen weitgehend unerforschten technisch-administrativen Aspekt der Vertragsanwendung zu lenken und einen Überblick über die zahlreichen durch den Vertrag geschaffenen Kommissionen und ihre Tätigkeit $\mathrm{zu}$ geben, die sich ebenso unterschiedlich darstellten wie die ihnen zugrunde liegenden Regelungsgebiete. Die Bandbreite reichte hierbei von „typischen“ Einrichtungen wie den Grenzkommissionen über die Ausschüsse zur Ausführung und Überwachung der Abrüstungsbestimmungen oder der für Kärnten angeordneten Volksabstimmung bis hin zu den Sachverständigenausschüssen für bestimmte Eisenbahnfragen und dem Novum der Reparationskommission.

Daraufhin füllten Walter Ibers (Graz) Ausführungen zum „Rettungsschirm“ für Österreich in Form der beiden „Völkerbundanleihen“ 1922 und 1932 den regelmäßigen Verweis auf die wirtschaftlichen Folgen des Vertrags mit Leben. Der Vortragende betonte, dass diese Anleihen keine unmittelbare Konsequenz des Vertrags darstellten und auch die im Vertrag enthaltenen Bestimmungen etwa zum Generalpfandrecht oder der Liquidation der österreichisch-ungarischen Bank lediglich den Rahmen für die österreichische Finanz- und Wirtschaftspolitik bildeten, deren Fehler - strategischer, psychologischer oder schlicht dilettantischer Natur - das ihre zur Situation beitrugen. Schließlich sollten (jeweils unter der Ägide eines niederländischen Kommissärs) entscheidende Reformen durchgeführt werden, die zu einer finanziell erfolgreichen Sanierung führen sollten, wirtschaftlich jedoch schwer zu bewältigen waren.

Nach einer Pause galt die Aufmerksamkeit dem bereits in den vorhergehenden Diskussionen immer wieder tangierten Thema der militärischen Bestimmungen. Tamara Scheer (Wien) wählte zur Verdeutlichung des (deutsch-)österreichischen Standpunkts einen internen Bericht aus den Unterlagen der Friedensdelegation, die in militärischen Fragen von Wien aus von einem Expertengremium, darunter Oberst Schneller und Theodor Körner, unterstützt wurden. Sie bemängelten am Vertragsentwurf vor allem, dass die schon bestehende internationale Liquidationskommission ignoriert, den Bestimmungen keine genaue Recherche vorangegangen, die Regelungen zur Militärluftfahrt eins zu eins vom deutschen Vertrag (der auf gänzlich anderen Voraussetzungen gründete) übernommen worden sei, Deutschösterreichs Wehrlosigkeit eine Bedrohung für den Frieden darstellen würde und ein Verbot des Milizsystems die Gefahr von 
Söldnertruppen nach sich zöge. Sachlich begründet oder nicht - in Bezug auf die militärischen Bestimmungen konnte die Delegation keine wesentlichen Veränderungen erreichen.

Auf die Ausführungen zur militärische Liquidation folgte der Vortrag Bernadette Biedermanns (Graz) zur Liquidation auf kulturellem Gebiet, d.h. der Aufteilung und Rückerstattung der Kulturgüter der ehemaligen Monarchie. Ausgehend von einigen Fallbeispielen erhielten die Teilnehmenden Einsicht in den theoretischen Hintergrund und die Herangehensweise der Provenienzforschung, bevor näher auf spezielle Rückforderungen der Tschechoslowakei und Belgiens und ein Beispiel bzgl. Italiens eingegangen wurde. Die Vertragsbestimmungen weisen Parallelitäten zur Aufteilung der Immobilien auf und die Anwendung des Territorialitätsprinzips für den Zeitraum nach 1914 zeugt von einer pragmatischen Entscheidung. Die Aufteilung der Kulturgüter und die Rückgabeforderungen zählen zu den am geringsten aufgearbeiteten Bereichen im Zusammenhang mit dem Staatsvertrag von St. Germain, sodass noch viele offene Fragen bestehen.

Ein kaum besser vertrautes Thema stellt die Ausgestaltung und Neuausrichtung des wirtschaftlich bedeutenden Bereichs des (Eisenbahn-)Verkehrswesens am Beginn der Zwischenkriegszeit dar. Nikolaus Reisinger (Graz) übernahm es, die historischen Entwicklungslinien nachzuzeichnen und wies darauf hin, dass auch in diesem Bereich die mentalitätshistorische Sicht nicht außer Acht gelassen werden könne. Wie schon im finanziellen Bereich galt auch hier, dass die Verluste aufgrund der einschneidenden Vertragsbestimmungen die Situation erschwerten (1919 waren manche Verbindungen gänzlich eingestellt, teilweise sollte sogar der Verbleib von ganzen Waggons ungeklärt bleiben), die Misere jedoch nicht ausschließlich darin begründet lag; zahlreiche technische Probleme wurden schon aus der Zeit der Monarchie mitübernommen. Hervorgehoben wurde auch das Interesse der tschechoslowakischen Politik auf diesem Gebiet, nachdem sich die Tschechoslowakei zum Ziel gesetzt hatte, zur neuen Eisenbahndrehscheibe Mitteleuropas zu werden.

\section{Die Pariser Nachkriegsordnung und die Verliererstaaten}

Nach diesem Fokus auf einzelne Themengebiete im Zusammenhang mit der österreichischen Regelung wechselte in diesem Panel die Perspektive wieder auf die gesamtvertragliche Ebene mit Blick auf einzelne Staaten. Nun richtete sich das Augenmerk auf die Vertragspartner der anderen Vororteverträge, wobei sich alle Vortragenden weniger auf einzelne Schwerpunkte konzentrierten, sondern erfolgreich um eine möglichst umfassende Darstellung bemühten.

Am Beginn standen zwei Vorträge zum Versailler Vertrag, dessen Regelungen in weiten Teilen als Vorbild für die übrigen Verträge gedient hatten. Zunächst analysierte Klaus Schwabe (Aachen) die Wahrnehmung und Folgen des Vertrages anhand der Ziele der USA, Frankreichs und Großbritanniens und der deutschen Erwartungen. Er betonte, dass Wilson nicht nur Ideologe war, sondern auch konkrete Vorstellungen für das von ihm angestrebte Ziel der kollektiven Sicherheit durch einen globalen, wissenschaftlichen Frieden hatte, während für Frankreich in erster Linie der Sicherheitsaspekt im Vordergrund stand und Großbritannien an einem Erhalt des (wirtschaftlichen) Gleichgewichts und einer Abgrenzung gegen das bolschewistische Russland interessiert war. Aus deutscher Sicht schienen Wilsons 14 Punkte einen Schutz vor den Interessen 
der Alliierten zu versprechen und die Möglichkeit eines Ausgleichsfriedens zu eröffnen. Das Vertragswerk trägt daher insgesamt dem Kompromissgedanken Rechnung, untereinander wie auch in Bezug auf den Kriegsgegner, blieb jedoch aus deutscher Sicht ein Anathema, unvereinbar mit der übernommenen moralischen Perspektive „eines demokratischen Rechtsfriedens“. Ohne diesen „Krieg in den Köpfen" (Krumeich) hätte sich die Verwirklichung angesichts der Flexibilität und der grundsätzlichen Annehmbarkeit zahlreicher Bestimmungen - allenfalls unter Ausklammerung bestimmter Themen - ganz anders darstellen können.

In eine ähnliche Richtung wiesen auch die Ausführungen von Hans-Christof Kraus (Passau), der sich den Folgen des Vertrags für die deutsche Außenpolitik widmete und konstatierte, dass mittlerweile von vielen der - zum Teil bis in die 2. Republik hineinreichenden - Paradigmen als völlig obsolet Abstand genommen wurde. Es stellt sich die Frage, welche Voraussetzungen es im neuen Kontext gab und welche Folgerungen die Politiker daraus zogen. Im Vordergrund sollte der Vorwurf des Art 231, die Reparationszahlungen, die Gebietsverluste und die als demütigend empfundenen Entwaffnungsbestimmungen stehen, während die neue Lage und die neuen außenpolitischen Möglichkeiten im Gesamtsystem übersehen wurden, die sehr wohl Handlungsspielräume eröffneten. Auch hier zeigt der Blick auf die einzelnen Siegermächte die Unterschiede. Wesentlich war neben der Nichtbeteiligung Russlands und der psychologischen Wirkung des Völkerbundbeitritts auch die letztendlich ausbleibende Ratifikation der USA, die in Deutschland bald einen künftigen Wirtschafts- und Handelspartner sehen und zu seinem größten Kreditgeber avancieren sollten.

Auf den Versailler Vertrag, der nach wie vor als bedeutend wahrgenommen wird, aber in erster Linie von historischem Interesse ist, folgte einen Überblick über den Vertrag von Trianon und dessen Stellenwert in der nationalen Erinnerung Ungarns. Ibolya Murber (Budapest) schilderte kurz die schwierigen Friedensverhandlungen, nachdem die wesentlichen Entscheidungen bereits endgültig vor der Teilnahme der ungarischen Delegation getroffen worden waren, wobei die Entente großes Interesse daran hatte, welche Regierung letzten Endes in Ungarn an die Macht kommen sollte. Die Auswirkungen des Vertrags, insbesondere die territorialen Verluste, sind bis heute prägend für die ungarische Erinnerungskultur und fixer Bestandteil der (Aus-)bildung; kritisch hervorgehoben wurde die bewusste politische Emotionalisierung.

Ebenfalls als eines besonders unglücklichen Kapitels wird des Vertrags von Neuilly in Bulgarien gedacht. Roumiana Preshlenova (Sofia) gab Einblick in diese „zweite nationale Katastrophe“ nach den Gebietsverlusten infolge des 2. Balkankriegs. Unter anderem verlor es 10\% seines Territoriums, 30\% der Bevölkerung und wurde ebenfalls zu Reparationsleistungen verpflichtet. Auch hier sind die innenpolitischen Konsequenzen von Bedeutung: Nachdem die Regierung aus Protest gegen die Friedensbedingungen zurückgetreten war, unterschrieb Stambolijski als neuer Ministerpräsident und machte daraus Karriere. Als Teil des Vertrags von Neuilly wird in der bulgarischen Geschichtsschreibung zudem das Abkommen zum „freiwilligen Bevölkerungsaustausch“ mit Griechenland betrachtet, das jedenfalls nicht zur Vereinfachung der Situation der Minderheiten führte.

Im Anschluss an die beiden Vorträge entspann sich trotz der vorgerückten Stunde die wohl lebhafteste Diskussion der Tagung, in deren Zentrum der Bevölkerungsaustausch stand, 
insbesondere inwiefern dieser dem Gedanken der ethnischen Säuberung Rechnung tragen sollte, wobei der Begriff der „ethnischen Säuberung“ selbst als Euphemismus kritisiert wurde.

Die ersten beiden Vorträge des Samstagvormittags richteten ihren Blick auf Südosteuropa und die Liquidation des Ottomanischen Reiches. Gingen bereits bei anderen Vorträgen das Thema des jeweiligen Vertrages mit völker- und verfassungsrechtlichen Fragen der Staatennachfolge Hand in Hand, so gilt dies besonders in Bezug auf die Verträge von Sèvres und Lausanne. Hüseyin Çiçek (Erlangen-Nürnberg) schilderte die Entwicklungen bis hin zur Entstehung des türkischen Staates und betonte das neue Selbstverständnis als türkische Nation, das sich durch die Regelungen zu den Minderheiten bedroht sah. Bis heute wird der Vertrag von Sèvres in der Türkei politisch instrumentalisiert und gilt als Ausdruck einer Geschichtskonstruktion und eines Apartheitsystems; die Archive sind verschlossen. Auch auf diesen Vortrag folgte eine ausführliche Diskussion, die von der Rolle der Briten über die Bedeutung des Scheiterns des Vertrages für die Nationalkonservativen in Deutschland bis hin zur Frage, wer denn nun tatsächlich Vertragspartei war, reichte.

Athanassios Pitsoulis (Hildesheim) rief zu Beginn seiner Präsentation ebenfalls die politische Aktualität des Vertrags von Lausanne in Erinnerung, der in den Grenzregelungen und militärischen Bestimmungen Klauseln enthält, über die sich Griechenland und die Türkei bis heute streiten; den Schwerpunkt seines Vortrags bildete der Bevölkerungsaustausch zwischen den beiden Staaten, der mittlerweile von der griechischen Literatur gegenüber der bisher positiven Bewertung realistischer zu behandeln versucht wird, während sich nur spärlich türkische Literatur zu dem Thema finden lässt. Der Vortragende selbst schloss eine positive Bewertung dezidiert aus: Vor allem die völkerrechtlichen Konsequenzen waren fatal, indem durch diese Vereinbarung demonstriert wurde, wie Zwangsvertreibungen nachträglich vertraglich legitimiert werden können; nicht umsonst sollten die Regelungen als Vorbild für das deutsch-italienische Abkommen von 1939 dienen.

\section{Folgen, Perzeptionen und Reaktionen}

Das letzte Panel bildeten zwei Präsentationen zu gänzlich verschiedenen, wenig bekannten Themen im Zusammenhang mit den Pariser Vororteverträgen, der Rolle und Perspektive des Vatikans in Paris und den technischen Aspekten der Grenzfestlegung, bevor die Tagung mit den völkerrechtlichen Konsequenzen des Vertrags und den Schlussworten Ziegerhofers ihren Abschluss fand.

Ausgehend von der „Friedensnote“, in der Papst Benedikt XV. unter anderem für die Macht des Rechts und die Einrichtung von Schiedsgerichten plädierte sowie den Verzicht auf die Reparationsforderungen und die Rückgabe der eroberten Gebiete gefordert hatte, gab Andreas Gottsmann (Rom) einen interessanten Einblick in die Politik des Vatikans im Hinblick auf die Pariser Friedenskonferenz, an der er trotz seiner Bemühungen im Vorfeld nicht durch Vertreter teilnehmen konnte. Die vom Vatikan verfolgte Strategie zielte auf die Etablierung des Papsttums als vermittelnde, übernationale, weltweit agierende Macht ab und markierte den vor allem durch Leo XIII. geprägten Übergang zum Streben nach ideologisch-geistlicher Weltherrschaft. Dem Ergebnis, den Friedensverträgen, stand der Vatikan - mit Ausnahme des Völkerbundes - insgesamt 
skeptisch gegenüber, da sie nicht auf christlichen Prinzipien beruhten; in Bezug auf Österreich, das er als wichtigen Faktor für die Stabilisierung Zentraleuropas wahrnahm, nahm der Vatikan zunächst eine abwartende Haltung ein, bevor Seipel sich um eine enge Allianz bemühen sollte.

Das Thema der Grenzziehung mag mit sämtlichen Pariser Vororteverträgen auf prominenteste Weise verknüpft sein, die tatsächliche Durchführung der Grenzbestimmungen und Festlegung der Grenzen führt jedoch ein Schattendasein. Aufbauend auf dem Gedanken der Universalität und Bedeutung von Grenzen erklärte der Geodät Heinz König (Wien) in seiner umfassenden Präsentation die technisch-praktische Seite der Festlegung von Staatsgrenzen und die Arbeit der Grenzkommissionen, bevor er den Teilnehmenden die Realisierung einiger Abschnitte der im Vertrag von Saint Germain getroffenen Grenzentscheidungen unter anderem anhand der Grenzurkunden anschaulich vor Augen führte.

Zu guter Letzt rückte der Vortrag Christian Pippans (Graz) über den Einfluss der Verträge auf die Entwicklung der Völkerrechtsordnung ein genuin rechtshistorisches Thema in den Mittelpunkt. Er konzentrierte sich dabei auf vier Schwerpunkte, die Neuerungen in Bezug auf das ius ad bellum sowie das ius in bello, den Minderheitenschutz und das Verhältnis der „qualifizierten Unabbängigkeit" zur klassischen Souveränitätsauffassung im Völkerrecht, wobei letzteres leider aufgrund der fortgeschrittenen Zeit nicht mehr behandelt werden konnte. Aus heutiger Sicht als besonders ungewöhnlich anzusehen ist unter anderem die mit den Verträgen gewählte Konstruktion einer Implementierung der autonomen Satzungen der ILO und des Völkerbunds, die mit ihrer Rechtspersönlichkeit als solche bereits eine entscheidende Neuerung gegenüber den Verwaltungsunionen darstellten und das Bild des institutionellen Völkerrechts zum Kooperationsrecht entscheidend verändern sollten, sowie die rasche Entwicklung (unter 30 Jahre) des Aggressionsverbots, während die individualrechtliche Ausgestaltung des Minderheitenschutzsystems auch für das geltende Recht typisch ist.

\section{Schluss}

Ziegerhofer sprach in ihrem kurzen Schlusswort allen Teilnehmern und vor allem auch dem von Wedrac geleiteten Organisationsteam ihren Dank für die erfolgreiche Veranstaltung aus, die wie wohl kaum eine andere die Bandbreite der in Paris getroffenen Regelungen aufzuzeigen vermocht hatte. Es wurde deutlich, dass zahlreiche Bereiche von der Forschung bis heute nicht vollständig aufgearbeitet wurden und in Anbetracht der großen Umwälzungen und des Umfangs der Konferenz selbst ins Hintertreffen gerieten. Insofern erwies sich die Wahl des Ausgangspunkts nicht der berühmt-berüchtigte Versailler Vertrag, sondern der unbekanntere Vertrag von Saint Germain - in Verbindung mit der nicht rein nationalen, sondern vielmehr gesamteuropäischen Perspektive, als glücklich. Dank gebührt den Veranstaltern für die reibungslose Organisation und den angenehmen, freundlichen Rahmen, der den Vortragenden zudem im Wege eines gemeinsamen Abendessens eine weitere Gelegenheit zu Vernetzung und Austausch bot. Es ist zu hoffen, dass gelebte Interdisziplinarität und internationale Zusammenarbeit auch in naher Zukunft dazu beitragen werden, mehr Licht auf dieses Kapitel unserer Geschichte zu werfen. 